\title{
Encrypted Network Traffic Classification and Resource Allocation with Deep Learning in Software Defined Network
}

\author{
Roy Setiawan ${ }^{1} \cdot$ Ramakoteswara Rao Ganga $^{2} \cdot$ Priya Velayutham $^{3}$. \\ Kumaravel Thangavel ${ }^{4} \cdot$ Dilip Kumar Sharma $^{5} \cdot$ Regin Rajan $^{6}$. \\ Sujatha Krishnamoorthy ${ }^{7} \cdot$ Sudhakar Sengan $^{8}$
}

Accepted: 15 March 2021

(c) The Author(s), under exclusive licence to Springer Science+Business Media, LLC, part of Springer Nature 2021

\begin{abstract}
The climate has changed absolutely in every area in just a few years as digitized, making high-speed internet service a significant need in the future. Future Internet is supposed to face exponential growth in traffic, and highly complicated infrastructure, threatening to make conventional NTC approaches unreliable and even counterproductive. In recent days, AI Stimulated state-of-the-art breakthroughs with the ability to tackle extensive and multifarious challenges, and the network community is initiated by considering the NTC prototype from legacy rule-based towards a novel AI-based. Design and execution are applied to interdisciplinary become more essential. A smart home network supports various applications and smart devices within the proposed work, including e-health devices, regular computing devices, and home automation devices. Many devices accessible through the Internet by Home GateWay for Congestion (HGC) in a smart home. Throughout this paper, a Software-Defined Network Home GateWay for Congestion (SDNHGC) architecture for improved management of remote smart home networks and protection of the significant network's SDN controller. It enables effective network capacity regulation, focused on realtime traffic analysis and core network resource allocation. It cannot control the Network in dispersed smart homes. Our innovative SDNHGC expands power across the connectivity network, a smart home network enabling improved end-to-end monitoring of networks. The planned SDNHGC directly will gain centralized device identification by classifying traffic through a smart home network. Several of the current traffic classifications approach, checking deep packets, cannot have this real-time device knowledge for encrypted data to solve this issue.
\end{abstract}

Keywords Software-defined network $\cdot$ Traffic detection $\cdot$ Security $\cdot$ Deep learning $\cdot$ Data flow

Ramakoteswara Rao Ganga

grkraoganga@gmail.com

Priya Velayutham

priya.saravanaraja@gmail.com

Sudhakar Sengan

sudhasengan@gmail.com

Extended author information available on the last page of the article 


\section{Introduction}

The SDN is a modern concept for computers and telecommunications networks. SDN's main objective is to tackle challenges that exist in IP-based networks, like complex management. With the present networks, operators will make several daunting modifications to network settings in the case of a small shift of network policy, regulations or topology, evaluating different protocols to provide fluid network security [1]. As a holistic definition, SDN distinguishes the data plan in control of transmitting data packets, and the control plan is responsible for the traffic engineering, management, and routing policies to address today's limitations and complexities of networking. Open Flow (OF) protocol is the most essential and functional communication protocol that allows the controller to communicate with the network switches [2]. Whereas, this protocol is a regular GUI mainly used within SDN. The OF switches provide one or two flow-inputs from the flow table. Every entry is made up of combined procedures and steps. The monitor leaves the tables up. Where the procedure consists of fields relating to the data from headers, like IP and MAC addresses of source and destination, port numbers, and also it needs other necessary details [3] [25]. Every behaviour specifies orders that are to be followed on the packet to fit the rule of entry.

Separating the data plan and control process allows network managers the opportunity to create programmable rules and monitor data plans with ease via the device. SDN even allows it easier to handle, customize, troubleshoot, and check new protocols and concepts in the network without any issues [4]. The proposed new deep learning model for SDN to identify traffic in data transmission to avoid congestions. Traffic detection is a significant case of network management as it has high flexibility and performance.

Methods exist for implementing the description of traffic in networks.

- They were identifying network and service layer interfaces by utilizing port numbers. Those approaches are not always practical, however.

- Deep Inspection Packets (DPI) were included. Such approaches are incredibly reliable, but some issues are there with their implementation because dynamic ports and authenticated traffic have not been allowed in current networks. This makes the bulky device overhead and infringes consumer privacy.

- While transmitting data from source to destination node data packet facing lots of troubles in choosing path to reach at right time.

- Selecting right node through dynamic allocation of node by deep learning based SDN model.

These approaches have their challenges, and recent work has concentrated on machine learning strategies that take advantage of traffic detection statistical properties [5]. While there are several difficulties for traffic classification in current networks, SDN's global definition of controllers enhances network management because it is quick and more comfortable collecting statistical details from network traffic switches.

Geographically dispersed data centres [6][6] are also referred to as Internet Data Centers (IDCs), and the cloud is used as a generic concept relating to infrastructure, applications, and resource aggregation within an IDC. An IDC usually consumes more megawatts of power and enforces high costs on electricity operators. Researchers have suggested load-aware server provisioning schemes to minimize energy costs, in that number of active mode servers is dynamically regulated depending on the fee. When the load is small, it is 
possible to program different sleeping mode servers [8]. Obtaining details on the amount of traffic in this model is a difficult problem. A system of technologies that guarantee service efficiency with the lesser cost for dispersed clouds is considerate traffic dynamics on both broad and small-time scales.

Our solutions leverage joint server provisioning for more time scales with cross-IDC load shifting for low time scales, as like flood control. We're addressing how cross-IDC load shifting will be configured to efficiently raising traffic complexity problems in a short time scale and cost-effectively.

Figure 1 indicates a view of the classification of traffic using a deep learning model. The article reviews the state-of-the-art deep learning methods for traffic classification in the networking environment described by software. There is a planned high-level overview of Software Defined Networking. The models of deep learning are then explored. Finally, an insight into the techniques of traffic classification is offered.

The organization of the article consists of following sections. The Sect. 2 consist of related works about various methods of recent works and Sect. 3 composed of proposed SDNHGC model. Data preprossing is described in Sect. 4 and supervised methods are explained in Sect. 5. Section 6 gives the performance evaluation of the proposed model and conclusion are well explained in Sect. 7.

Fig. 1 Flow of proposed SDNHGC model

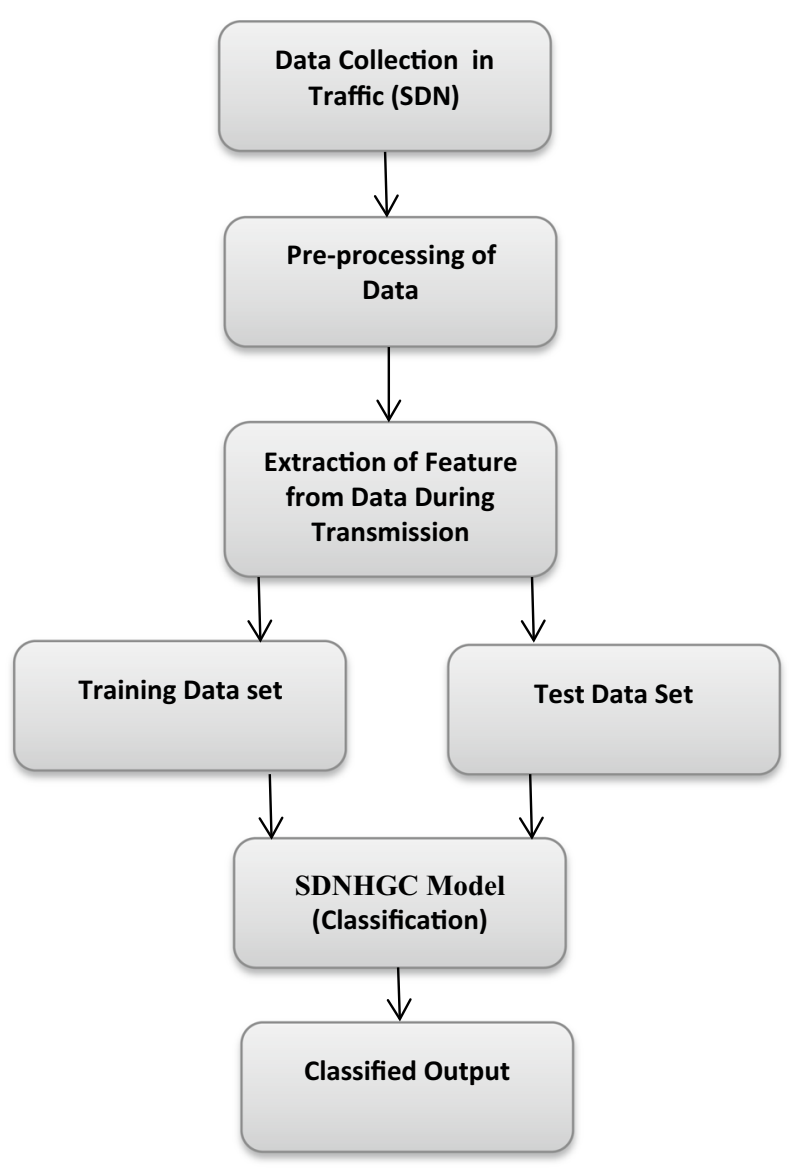




\section{Related Works}

The various types of congestion are focused on IP addresses and deep packet inspection. (DPI) [9]. In applications where IP-based Classification cannot be used, changing IPs are used. In the particular circumstance of DPI, the sequence is classified for the query. For problem-solving, search queries have been used. With the unexpected progress in systems, the sequence identification of a wide range of applications is heavily restricted. The DPI system could not categorize encrypted communications.

The article mentions the performance of multiple apps based on the technique of deep learning. The crowdsourcing method is being used to gather preliminary information from real data. Policy decisions are attempted on the central authority SDN channel. Network management is suggested with a perspective on the combined optimization process for the use of resource management. The AI [10] methodologies for handling the Spatio-temporal alterations in the router are addressed. This phase represents the application of Artificial Intelligence (AI) by implies SDN for the controller's performance prediction. The characteristics used are round time, outcome, and the contents of the virtual machine. A method is used to measure the square difference between the actual and predict SDN efficiency. In classify the traffic of the network, machine training and software-based system architectures have been used. The findings are controlled and reliable learning. Classification methods used in spam detection for cybersecurity are the K- Nearest Neighbours (K-NN) [11], the random forest classification, and the Decision Tree.

Investigators developed a framework to determine the implementation of various wireless communication flow conditions with several mobile devices linked to an OF switch [12]. A machine-based trainer will be required to provide information in the control program. On the other hand, the OF switch receives the properties of various flows and forwards them to the control network to produce a method used to recognize a single layer on those kinds of flow conditions. When a server is invented, the OF switch exchanges properties, depending on a machine learning approach, to the traffic classification algorithm. The use of the source flow would then be evaluated. The system is predicated on the method C5.0 of choice.

SDN [13] is supported by the empirical flow control precision so that it does not know the data flow's data storage information that makes SDN vulnerable to Trojan attacks, worms, spam, etc. Data packets must be discovered in the wireless applications required to preserve network security. The article suggested a computer simulation with a time-specific network inspired by methodological approaches to transmit epidemics around neighbourhoods in distributed patterns. The findings may help defense against spyware in the SDN control strategy to reduce cybersecurity instances and help avoid them feasibly.

A zero-sum game [16] is a game-theoretic principle and a non-cooperative game. Because its method is relatively straightforward, the attacker and the defender can build models with a close to zero-sum in unauthorized access or defense. The intruder has a significant outcome when he damages successfully, whereas the defender has a low rating, and the maximum of the two is void. Two very different attacks and defense resources are restricted in the network detection and mitigation model. The game model of the defence and attack is formed. We can improve our military capabilities, cause the risk of attack vectors, and offer a suitable packet methodological approach within the wireless applications through a quantitative analysis of nodes in the network and a correspondent profit value.

Applied to different problematic areas were Machine learning methodologies [17]. A distantly related domain consists of a large number of predictive metadata analytics known 
as distributed mining. Although ML techniques can then be used to assist knowledge discovery, the purpose of data removal problems is to analytically and identify essential data-their features, parameters, boundary conditions, frequency complexity, allocation of probability, and transformations. But Machine learning means going much further than data mining to predict activities or event process accurately.

The combined neural network and firefly are configured to be autonomous and reliable for boiler plants. Optimization algorithms were also used for wireless communication link adaptive responses to optimize efficient energy, considering QoS [18] constraints. To communicate with malicious code problems, QoS embraces controlling Neural traffic networks that are considered equal to suppress optimized intrusion in Sensor nodes. SDN based computer architecture is implemented for Open vSwitch and POX packages while transmitting data over the network. This produces QoS guaranteed services [24] along with analytical solutions.

\section{Proposed Methodology-SDNHGC}

A smart home network incorporates a range of connected appliances and technologies within the proposed research, including home control appliances, standard computing devices, [19], e-health devices, etc. Most of the devices access the Internet through HGW in a smart home. This paper suggests Software-Defined Network for congestion (SDNHGC) architecture to help handle dispersed smart home networks, and it assists the central network SDN Controller. It enables effective network capacity regulation [20], focused on the real-time traffic analysis and core network resource allocation. This cannot control networks in dispersed smart homes. Our SDNHGC plan expands power over the access network, connected home network, enabling the network's end-to-end control. The problems faced by the classification of payload dependent information, like encrypted software and the user data protection, Moore, and semi-supervised naive methods used to identify the network traffic dependent on the statistical flow of features.

We suggested classifying traffic for real-time in Fig. 2 with purposes based on the latest packets of a flood. We have extended the research by extending the Bayesian neural networks to correct analysis by traffic. Used unidirectional statistical tools in the network center for traffic sorting and recommended an algorithm with the potential to predict missing tools. We suggested using only the size of the first SSL link packets to identify the encrypted applications proposed to evaluate the randomness of message content generated by encryption processing by using Pearson's chi-Square test-based methodology. Fingerprints dependent protocol focused on Probability Density Function (PDF) to convey the three statistical properties of traffic compactly. Their function is expanded into a method of parametric optimization.

\section{Pre-processing the Data Packet}

The DataNet is at the center of the new HGC SDN system. Though, it captured raw data packet is not ideally stored (and developed) in DataNet form. For example, this research's packet data is in PCAP/PCAPNG format [21]. A basic kit includes details which do not include Classification, ex. Max TCP/UDP/ICMP Numbers etc. This segment presents the 


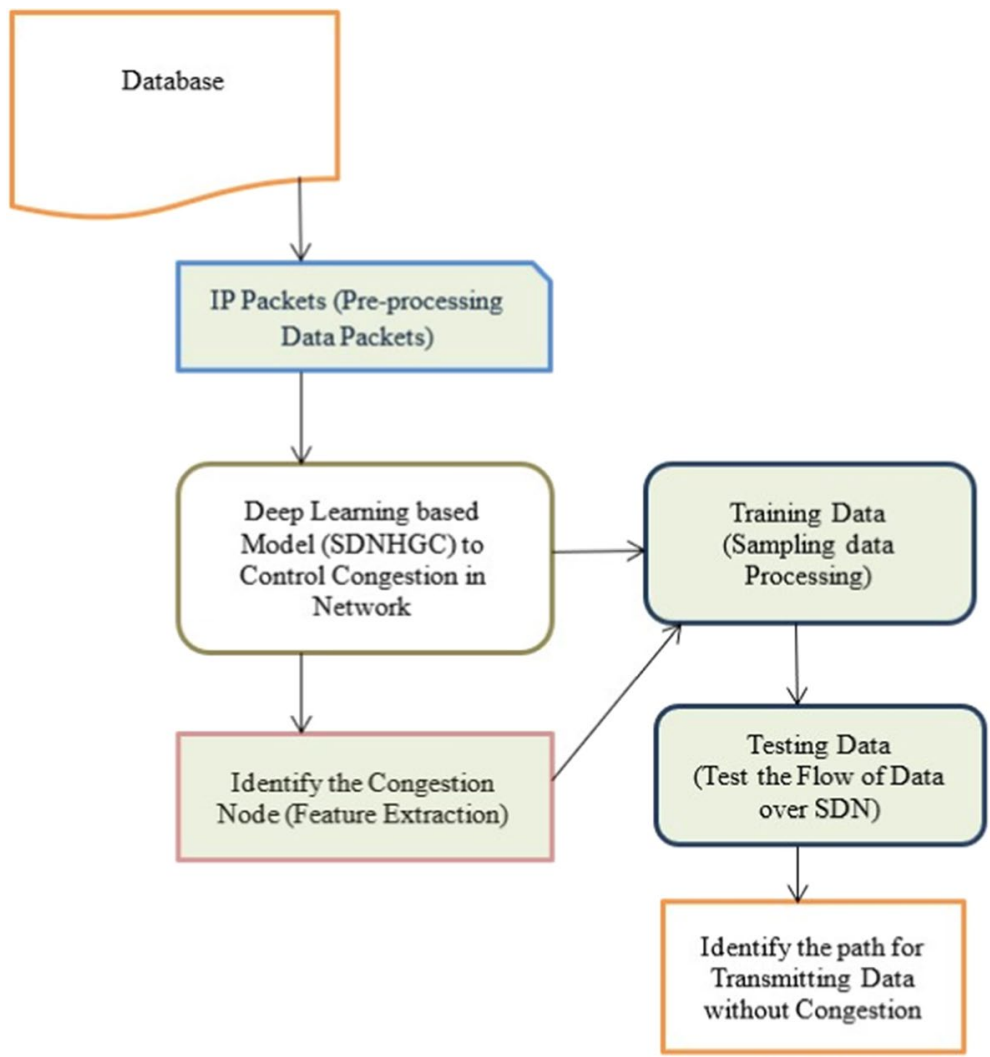

Fig. 2 System architecture of SDNHGC model

raw data kit and raw data collection pre-processing procedures for building DataNet under the training program and checking data packets at all SDNHGC.

\subsection{Pre-processing Packet Byte Vector}

There are three phases involved in pre-processing a raw data package: evaluating, cutting/ filling, and normalizing. In FIG, you can see a description of the pre-processing protocol. RAW Data Package is processed byte-by-byte, similar to an image pixel that will be easily imported into Classic deep learning. The Ethernet header is removed from a raw data packet in the study. Details on the data link layer like frame size, MAC Address, etc., is not helpful when classifying packets. The process of analyzing a packet reduces the input size. Additionally, some noise is screened during the Classification accuracy enhancement phase. When trimming and padding to zero, Packet input to the classifier is defined on the size of each data $x$. The proposed deep-learning-based kit classificatory needs an equivalent size of all inputs. Precisely specify as the target size for the DataNet entry, where $0<\mathrm{n} 1500$. The Main Transmitting Device is 1500 bytes in size. Depending on the duration when contrasted with n, an input packet is cut off or loaded with zeros. An entry is represented as Packet Byte Vector (PBV), after truncation and padding with zeros. 


\subsection{Pre-processing the Packet by Using Byte Matrix}

A dataset of the labelled raw data packets has been processed to build DataNet. Although the data aspect differs with the same program class itself, a regular size allows servicing and upgrading simpler, introducing more potential applications to the network. 1480 is chosen for the remainder of this work.

\subsection{Deep-Learning Based Encrypted Data Classifier}

' $\mathrm{X}$ ' is used to construct DataNet, after pre-processing; H. Crypted packet classifiers for the files. We build in particular three DataNet styles at home on Multi-Layer Perceptron (MLP), Convolutional Neural Network (CNN), and Stacked Auto Encoder (SAE).

\section{Supervised Method}

The supervised traffic classifiers to evaluate supervised training data and create an implied feature predicting the output class for any test flow. Adequate controlled teaching data is a familiar concept when classifying traffic under supervision. To fix the issues found in detecting payload information, such as authenticated apps and user details.

\subsection{Flow Correlation with Traffic Classification Method}

The present new framework that we can call for Traffic Classification uses short, Correlation Information/TCC. Here, A novel nonparametric method is offered to incorporate information on flow correlation effectively for the classification process. The controller is extended until the transport layer adjusts dynamically with an integrated multi-layer [22]. Figure 3, traffic is identified through the network and distributed over the node.

\subsection{Co-relation Analysis}

The same framework generates correlated flows that share the same three-tuple. For example, in a short time, multiple flows triggered by various hosts would all link to the same host in TCP port 80. Very possibly, these flows are created by the same application as a web browser. A payload dependent clustering approach for protocol inference was suggested in many functional traffic classification schemes to find the three-tuple heuristic for flow similarity, in which they clustered flows towards equivalence clusters [23] by using heuristic process. The accuracy of the heuristic three-tuple for the realworld traces has been verified. Figure 4 represents how the data packet is allocated for multiple paths to avoid traffic congestion from the source node. 


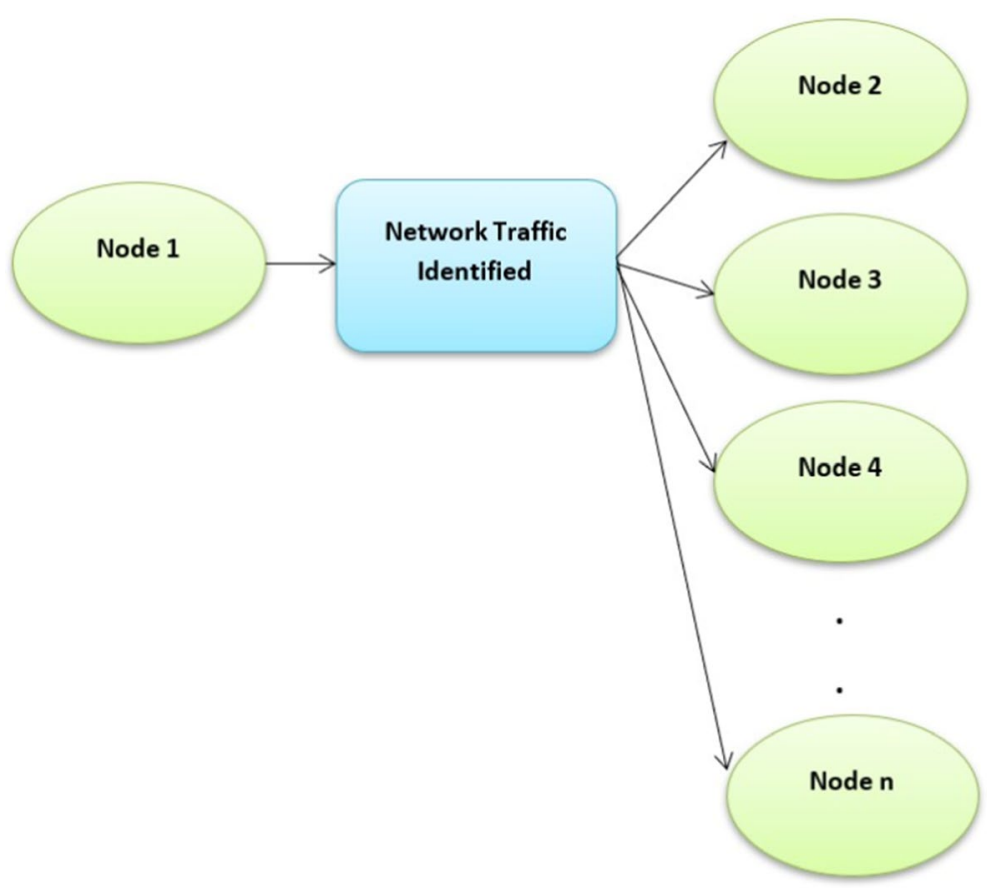

Fig. 3 Traffic classification diagram

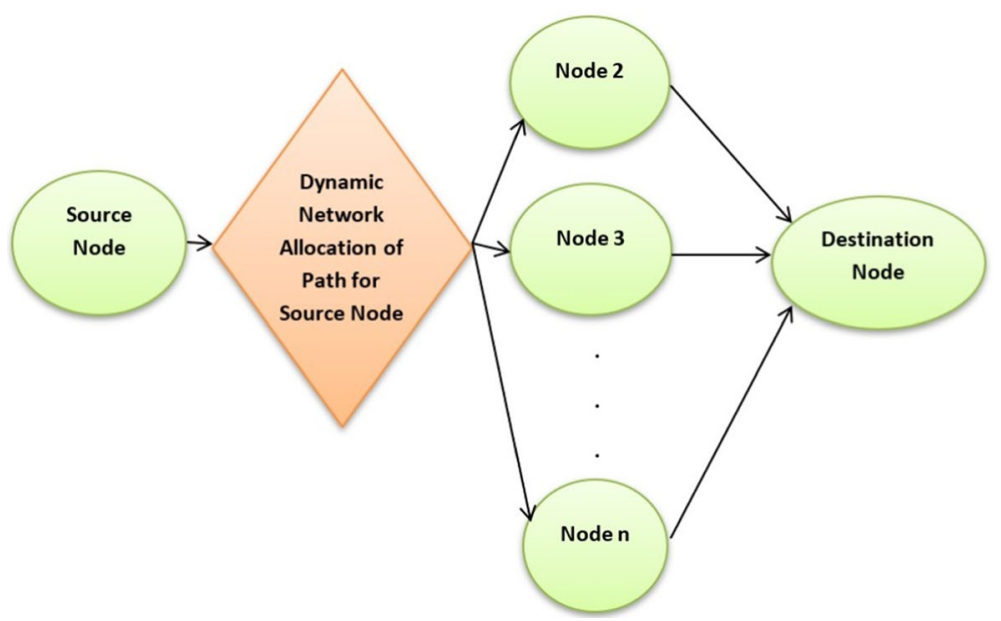

Fig. 4 Dynamic allocation diagram for network traffic

\section{Performance Evaluation}

The numerical efficiency requires time to understand, the sum of storage and time to identify. Second, the NN classifier doesn't require any learning mechanism associated with the approaches we suggest. Like SVM (Support Vector Machine) and neural nets, 
Fig. 5 Login module

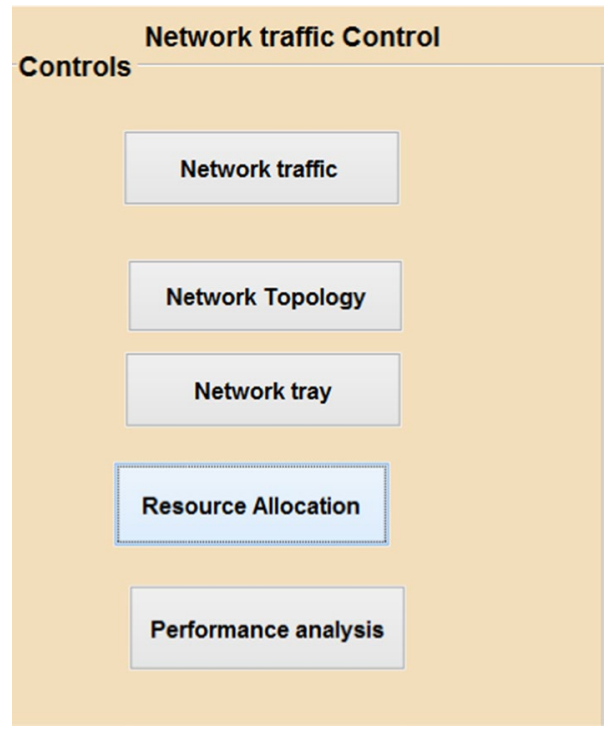

some other supervised methods require time for learning their classification model's parameters. Next, the methods proposed to use the nearest neighbour rule require data storage to train data samples. Where the amount of capacity is limited, even if the size

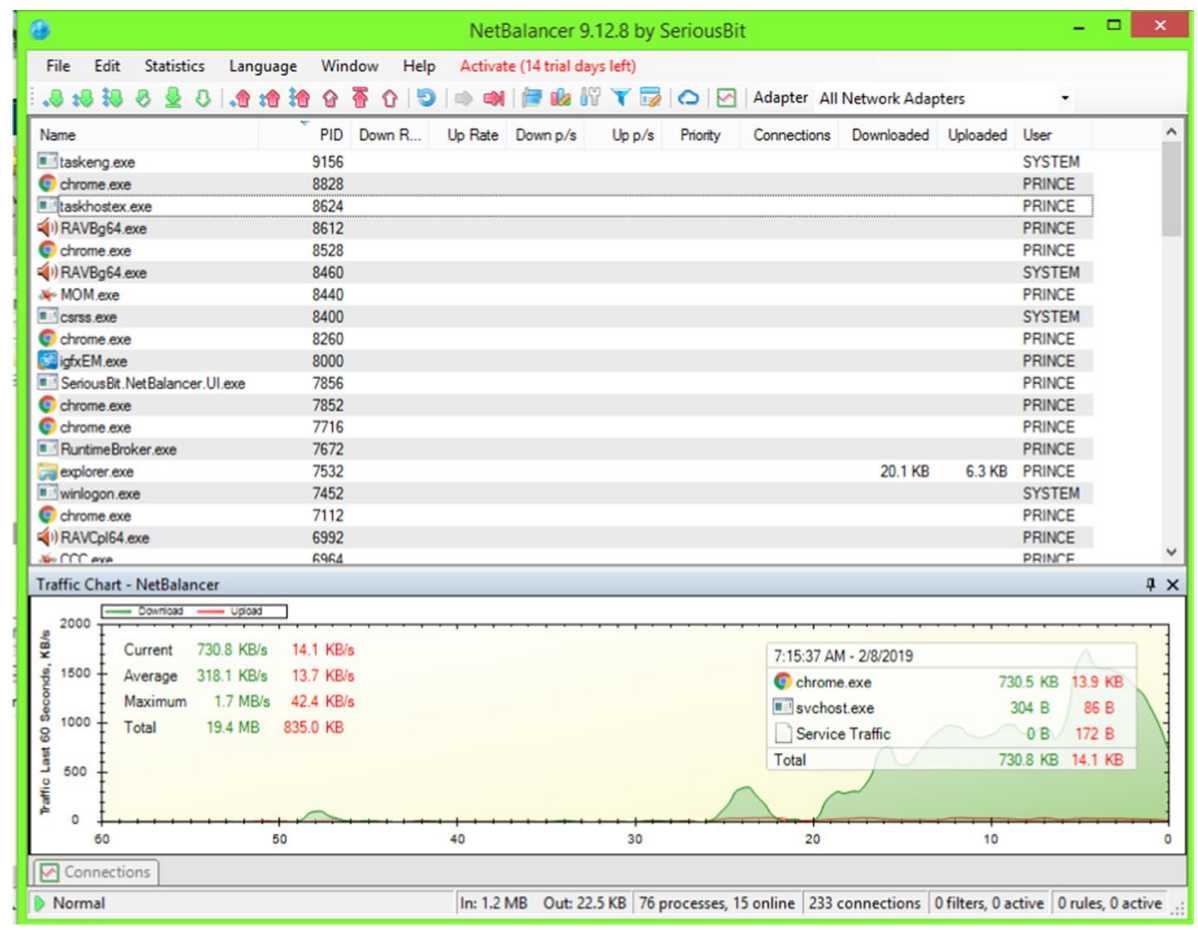

Fig. 6 Network traffic classification monitor module 
of the training data is limited. The performance is measured by allocating the resources and performance analyzer to avoid network traffic in SDN. This will be represented in Fig. 4 (Fig. 5).

Figure 6 shows how the network is controlled through SDN using NetBalancer by serious data transmission in the form bit identified with their PID (Product ID) value. While congestion occurs, the network manager allocates the data transmission path to the node using the network topology assigner in Fig. 7 through MATPLAN (MATlab and probability-based PLANning) tool. Here we used MATLAB for simulating the proposed work. The average packet length is measured by using following Eq. (1), Where, L represents the Length of data packet, ' $n$ ' gives the number of packets and k shows the data value of packet $P$,

$$
L^{n}=\sum_{k_{0}=0}^{n}\left(K_{0}+K_{1}\right) P\left(k_{0}, k_{1}\right)
$$

Figure 8 can identify which network node is busy, and the node is reallocated through this result automatically as the pending requests are detected and make data transfer through another node to allocate other resources for data transmission.

Figures 9, 10 and 11 demonstrations the performance analysis graph received in the MATLAB simulation tool and compared with the AI-NTC and new proposed SDNHGC deep learning models for plotting specificity and sensitivity. Equation (2) specifies sensitivity measurement by considering the data packet classification for transmitting through the node.

$$
\begin{aligned}
& \text { Sensitivity }=\frac{T P}{T P+F N} \\
& \text { Specificity }=\frac{T N}{T N+F P}
\end{aligned}
$$

Equation 3 produces the resulting specificity following the traffic classifiers.

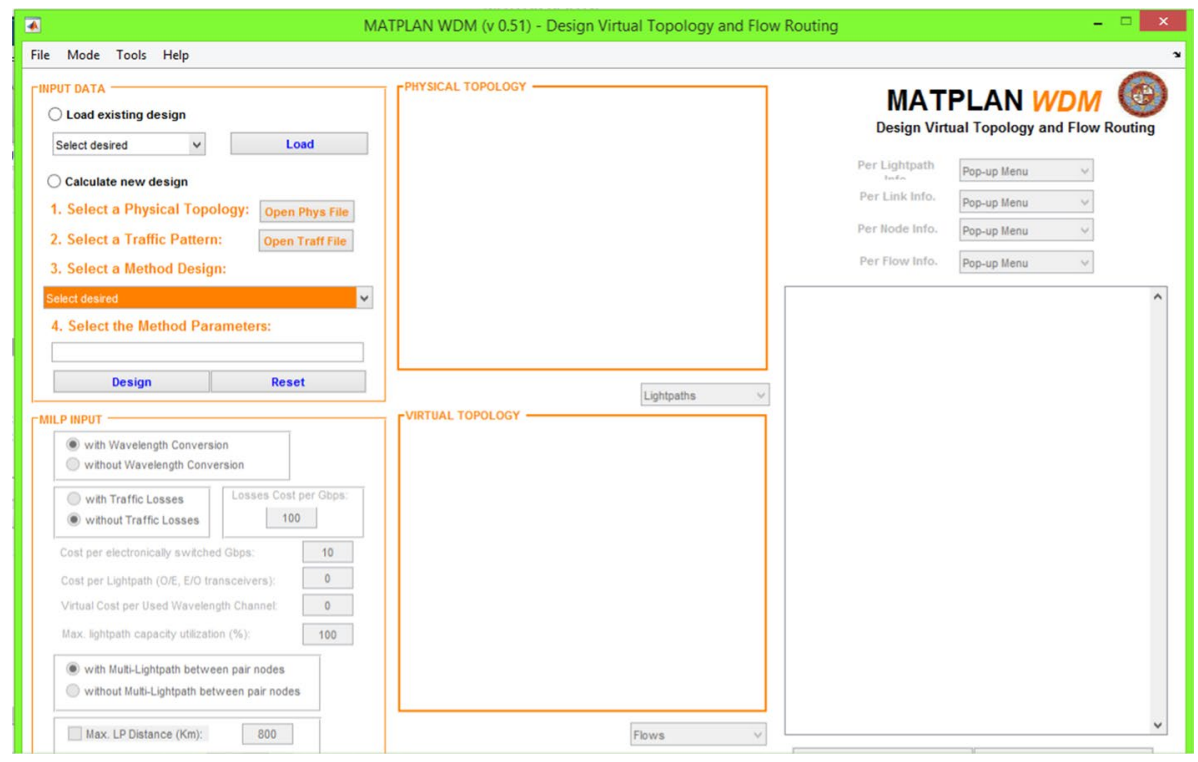

Fig. 7 Network topology assigner and traffic table viewer 

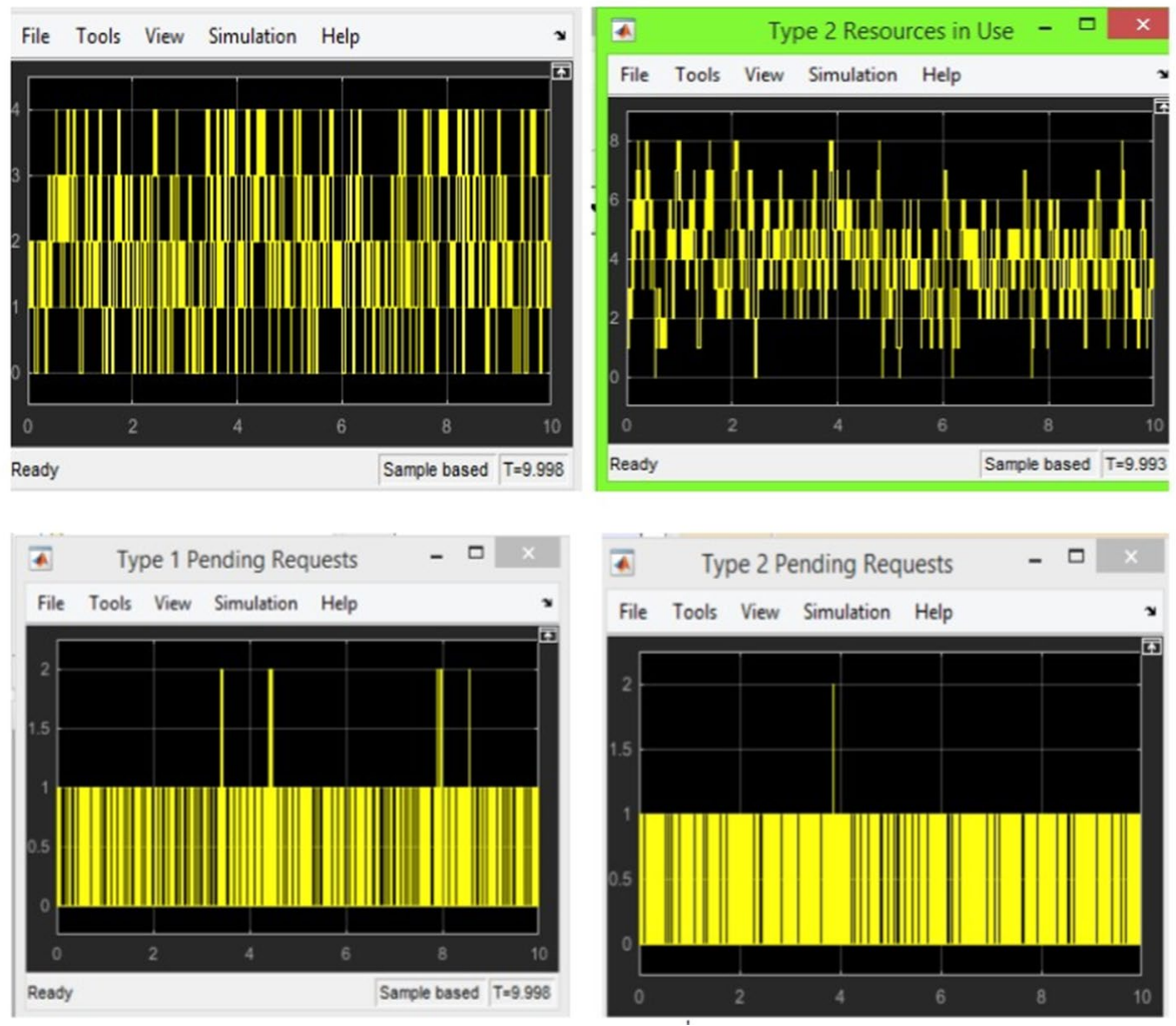

Fig. 8 Network resource allocation module

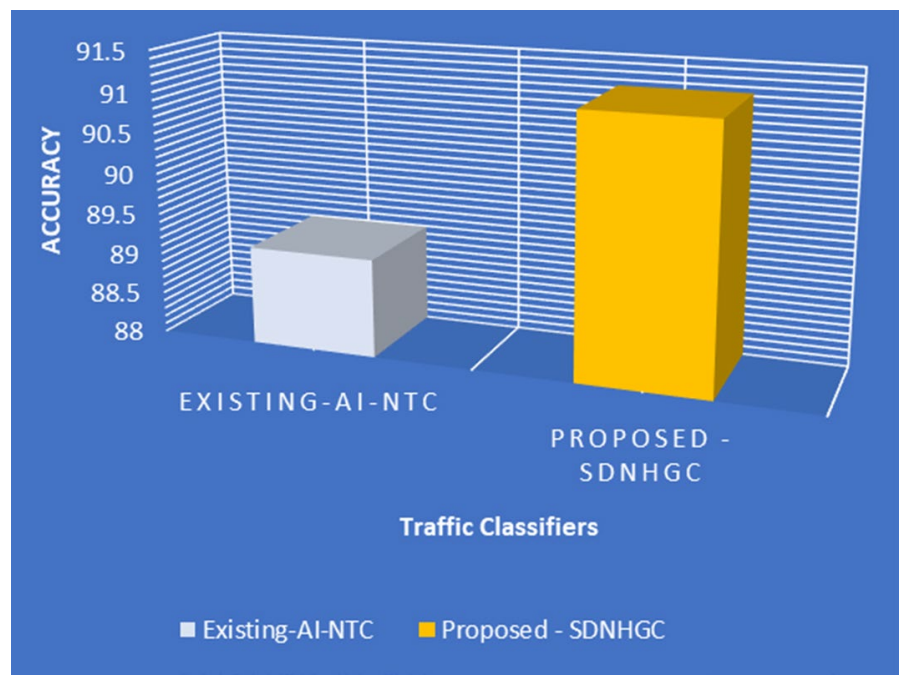

Fig. 9 Performance analysis of comparison graph 


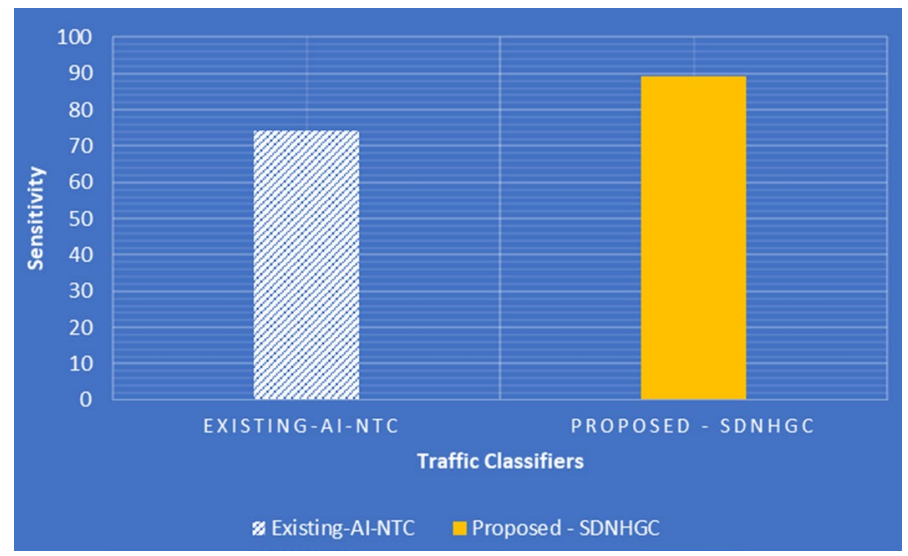

Fig. 10 Performance analysis of plot of sensitivity

Fig. 11 Performance analysis of plot of specificity

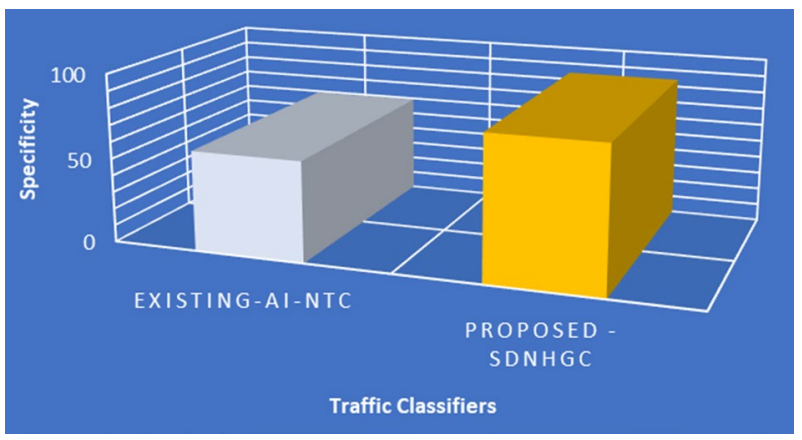

\section{Conclusion and Future Work}

In this article, a SDNHGC System is suggested to promote integrated end-to-end network processing and QoS management. DataNet deep learning is the cornerstone of the current HGW system, focused on encrypted data packet classifiers. The proposed DataNets was built utilizing three methods: SAE, MLP, and CNN. An open database of more than 20; The proposed DataNets were built and evaluated using packages from various applications. The experimental results revealed that the proposed SDNHGC platform and accurate packet detection and robust real-time computational power processing can be implemented in a smart home network with DataNets installed. Adapt them in future research to improve network capital management, make creative business strategies, etc., feasible without compromising the security/privacy of service providers or users. Here, discussion made for classifying traffic using very few controlled train samples. Thus, A novel nonparametric method, TCC, has been introduced to analyze and integrate connection knowledge in necessary traffic details into the traffic classification. This will provide a detailed program structure study and efficiency gains from theoretical and scientific viewpoints, which strongly endorse suggested solution. A variety of studies over two real-world traffic type of data sets demonstrate that traffic classification 
efficiency can be increased under the crucial circumstances of exceptionally few controlled training tests. The suggested solution will be used in a broad range of applications, like automated detection of the unknown applications through the network traffic collected and the semi-supervised data mining for the network packet process.

Funding Not applicable.

Availability of Data and Material Not applicable.

Code Availability Not applicable.

\section{Declarations}

Conflict of interest The authors declare that they have no conflicts of interest.

\section{References}

1. Cisco Global Cloud Index: Forecast and Methodology, 2012-2017, white paper, Cisco Systems (2013).

2. Xu, D., Liu, X., \& Fan, B. (2011). Minimizing energy cost for internet-scale datacenters with dynamic traffic. In Proceedings of the IEEE 19th international workshop on quality of service (IWQoS) (pp. $1-2)$.

3. Chen, G., He W, Liu, J., Nath, S., Rigas, L., Xiao, L., \& Zhao, F. (2008). Energy-aware server provisioning and load dispatching for connection intensive internet services. In Proceedings of the 5th USENIX symposium on networked systems design and implementation (NSDI 08) (pp. 337-350).

4. Malik, A., de Fréin, R., Al-Zeyadi, M., \& Andreu-Perez, J. (2020, July). Intelligent SDN traffic classification using deep learning: Deep-SDN. In IEEE explore, 2020 2nd international conference on computer communication and the internet (ICCCI). https://doi.org/10.1109/ICCCI49374.2020.9145971

5. $\mathrm{Xu}, \mathrm{H} .$, \& Li, B. (2015). Temperature aware workload management in geo-distributed datacenters. IEEE Transactions on Parallel and Distributed Systems, preprint. https://doi.org/10.1109/TPDS.2014. 2325836

6. Glanz, J. (2012). Power, pollution and the internet. New York Times, 22 Sept. 2012; www.nytimes Total costs (Dollar) DTJ queue delay (sec) Closely coupling cross-IDC DTJ load shifting to capacity allocation .com/2012/09/23/technology/data-centers-waste-vast-amounts-of-energy-belying-industryimage.html.

7. Tu, J., Lu, L., Chen, M., \& Sitaraman, R. K. (2013). Dynamic provisioning in next-generation data centers with on-site power production. In Proceedings of the 4th international conference on future energy systems (energy 13) (pp. 137-148).

8. Georgiadis, L., Neely, M. J., \& Tassiulas, L. (2006). Resource allocation and cross-layer control in wireless networks. Foundations and Trends in Networking, 1(1), 1-149.

9. Rao, L., Liu, X., Xie, L., \& Liu, W. (2010). Minimizing electricity cost: optimization of distributed internet data centers in a multi-electricity-market environment. In: Proceedings of the IEEE INFOCOM (pp. 1145-1153).

10. Tse, S., \& Choudhury, G. (2018, June). Real-time traffic management in AT\&T's SDN-enabled core IP/optical network. In IEEE Xplore, optical fiber communications conference and exposition (OFC).

11. Lin, M., Wierman, A., Andrew, L. L., \& Thereska, E. (2013). Dynamic right-sizing for power proportional data centers. IEEE/ACM Transactions on Networking, 21(5), 1378-1391.

12. Stanojevic, R., \& Shorten, R. (2010). Distributed dynamic speed scaling. In Proceedings of the IEEE INFOCOM (pp. 426-430).

13. Benson, T., Anand, A., Akella, A., \& Zhang, M. (2010). Understanding data center traffic characteristics. ACM SIGCOMM Computer Communication Review, 40(1), 92-99.

14. Chen, Y., Das, A., Qin, W., Sivasubramaniam, A., Wang, Q., \& Gautam, N. (2005). Managing server energy and operational costs in hosting centers. In Proceedings of the ACM SIGMETRICS international conference on measurement and modeling of computer systems ( $\mathrm{pp}$ 303-314). 
15. Morzhov, S. V., \& Nikitinskiy, M. A. (2018, March) Development and research of the pre-firewall network application for floodlight SDN controller. In Proceedings of the Moscow workshop electronic and networking technologies (MWENT) (pp. 1-4).

16. Hadi, F., Imran, M., Durad, M. H., \& Waris, M. (2018, Jan.). A simple security policy enforcement system for an institution using SDN controller. In Proceedings of 15th international Bhurban conference on applied sciences and technology (IBCAST) (pp. 489-494).

17. Xiong, Z., Zhang, Y., Niyato, D., Deng, R., Wang, P., \& Wang, L. (2019). Deep reinforcement learning for mobile $5 \mathrm{G}$ and beyond: Fundamentals, applications, and challenges. IEEE Vehicular Technology Magazine, 14(2), 44-52.

18. Witanto, J. N., \& Lim, H. (2019). Software-defined networking application with deep deterministic policy gradient (ICCMS 2019). ACM, New York, NY, USA (pp. 176-179). https://doi.org/10.1145/ 3307363.3307404

19. Rezaei, S., \& Liu, X. (2018). How to achieve high classification accuracy with just a few labels: A semi-supervised approach using sampled packets. arXiv preprint arXiv:1812.09761.

20. Koponen, T., Casado, M., Gude, N., Stribling, J., Poutievski, L., Zhu, M., Ramanathan, R., Iwata, Y., Inoue, H., Hama, T., \& Shenker, S. (2010). Onix: A distributed control platform for large scale production networks. In Proceedings of USENIX operating systems design and implementation (OSDI), Vancouver, BC, Canada.

21. Nawrocki, P., \& Sniezynski, B. (2020). Adaptive context-aware energy optimization for services on mobile devices with use of machine learning. Wireless Personal Communications, 115, 1839-1867. https://doi.org/10.1007/s11277-020-07657-9.

22. Zhou, Z., \& Niu, Y. (2020). An energy efficient clustering algorithm based on annulus division applied in wireless sensor networks. Wireless Personal Communications, 115, 2229-2241. https://doi.org/10. 1007/s11277-020-07679-3.

23. Rawat, P.S., Dimri, P., Kanrar, S., \& Saroha, G. P. (2020). Optimize task allocation in cloud environment based on big-bang big-crunch. Wireless Personal Communications, 115, 1711-1754. https://doi. org/10.1007/s11277-020-07651-1.

24. Yen, T.-C., \& Su, C.-S. (2014). An SDN-based cloud computing architecture and its mathematical model. IEEE (pp. 1728-1731).

25. Ganesh Kumar, K., \& Sudhkar, S. (2020). Improved network traffic by attacking denial of service to protect resource using Z-test based 4-Tier GeomarkTraceback (Z4TGT). Wireless Personal Communications, 114, 3541-3575. https://doi.org/10.1007/s11277-020-07546-1.

Publisher's Note Springer Nature remains neutral with regard to jurisdictional claims in published maps and institutional affiliations.

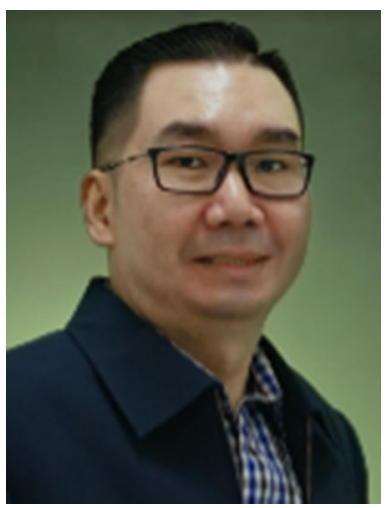

Roy Setiawan is a lecturer in the Department Management, Faculty Business, and Economic at Universitas Kristen Petra, Indonesia, with research interests in the field of Business and Management. He graduated from the informatics engineering and management science programs. He previously served as the Chairman of the Business Management Study Program and Head of the Leadership Laboratory at Universitas Kristen Petra. He is currently completing doctoral studies in Management at Universitas Airlangga Surabaya and is developing a new model in his research. 

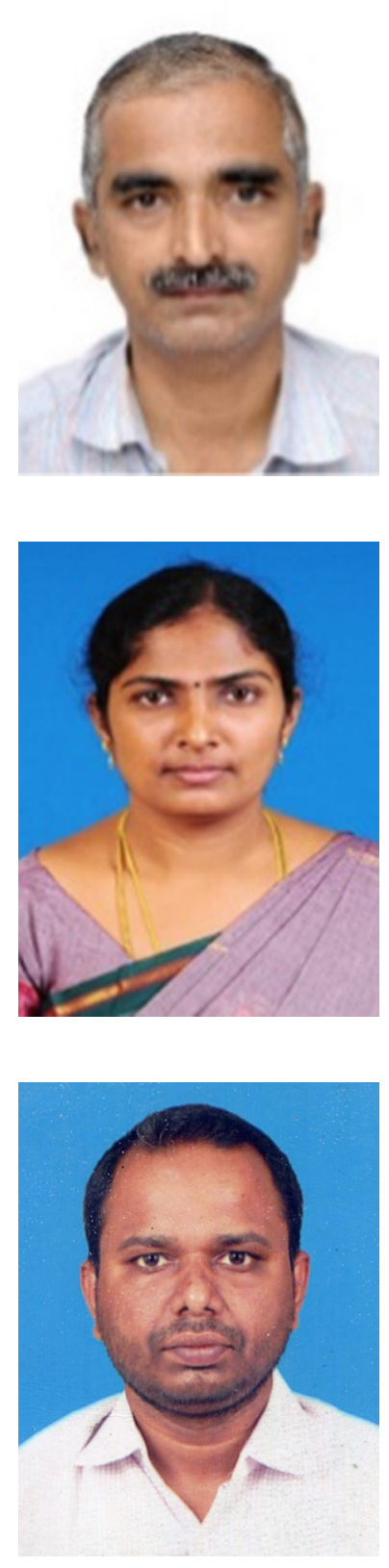

Ramakoteswara Rao Ganga is having $25+$ years of experience as a faculty member in Department of Computer Science. \& Engineering. Currently he is working as a Professor in Department of Information Technology at K.L. University. He is an active researcher in area of Cyber Security.

Priya Velayutham has received her Ph.D. degree in Information and Communication Engineering in 2017 at Anna University, Chennai. Currently, she is working as an Associate Professor in Computer Science and Engineering at Paavai Engineering College, Namakkal-637018, Tamil Nadu, India. She has more than 12 years of experience in Teaching and Research. Her research interests are in the areas of Artificial Intelligence, Machine Learning, Deep Learning, Cloud computing, Image processing, Data Science, Internet-of-Things and Bio-informatics. She published her research articles in reputed international journals, which is having a high impact factor. She received Best Faculty Award in Junior/ Department of CSE in 2019 from Shri P.K Das Memorial Best Faculty Award and Best Researcher Award in 2020 for her publications. She is a Life Member of the Indian Society for Technical Education (ISTE).

Kumaravel Thangavel received the BE degree in Computer Science and Engineering from PGP College of Engineering and Technology and ME Computer and Communication Engineering in SSN College of Engineering. He worked as an Assistant Professor (SRG) in Computer Science and Engineering Department in Kongu Engineering College, Perundurai, Tamil Nadu. His areas of interest Data Mining, Machine Learning and Deep Learning. 

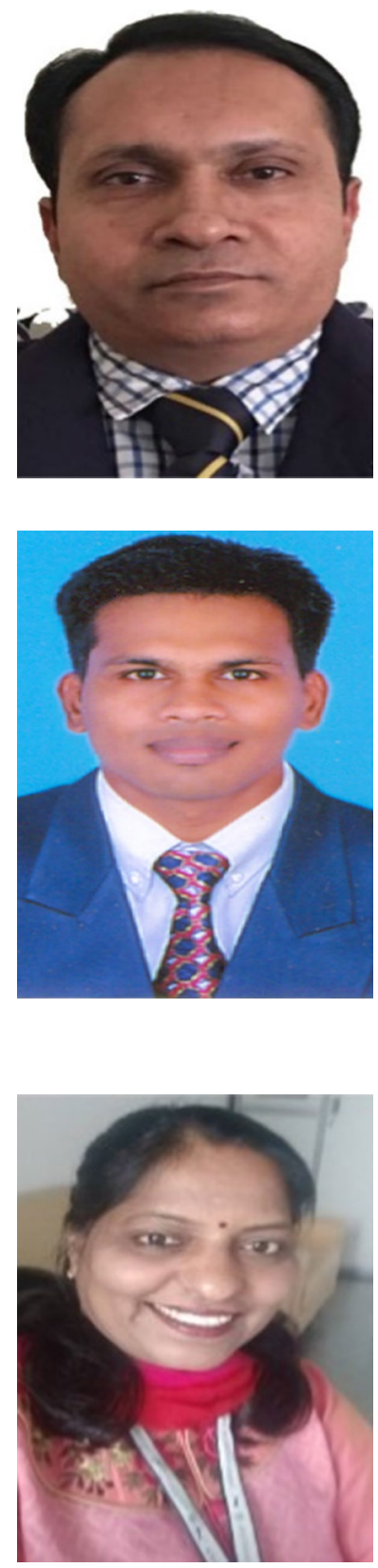

Dilip Kumar Sharma did his M.Sc. (Mathematics) from Government PG College Guna (M.P.) in the year 1998 and M.Tech. (Future studies and planning) from School of Future studies and planning, Devi Ahilya University Indore (M.P.) in the year 2002. He did his Ph.D. from JUIT Wakanaghat, Solan (H.P.) in the year 2009. Presently he is working in Jaypee University of Engineering and Technology, Guna (M.P.). He has about 16 years teaching experience. He is member of IEEE, Bombay Section. He is life member of Forum for Interdisciplinary Mathematics (FIM) and Indian Science Congress. He has published many research papers in reputed international journals and presented research papers in Conferences. He has visited NUS Singapore and Concordia University, Montreal, Canada. He is also a member of editorial board of the JUET Research Journal of Science and Technology. He has supervised 3 Ph.D. scholars and one Post-Doctoral fellow sponsored by NBHM, DAE, Mumbai and he is currently supervising two Ph.D. scholars.

Regin Rajan has received his B.E. (Computer Science and Engineering) degree in the year 2007 From CSI Institute of Technology, Nagercoil, and M.E. (Computer Science and Engineering) degree in the year 2011 from Annamalai University, Chidambaram. Currently he is pursuing his Ph.D. in Anna University, Chennai, in the field of WSN, VANET, and MANET. He has 9 years of teaching experience, and he is working as an Assistant Professor in the Department of Information Technology at Adhiyamaan College of Engineering, Hosur, Tamil Nadu.

Sujatha Krishnamoorthy is working as an Assistant professor in Department of Computer Science Kean Wenzhou University China and active member of CSI with 16 years of teaching experience. Her specialization is Digital image processing with Image fusion. She has published over 60 papers in International refereed journals like Springer and Elsevier. She has delivered several guest lectures, seminars and chaired a session for various Conferences. She is serving as a Reviewer and Editorial Board Member of many reputed Journals and acted as Session chair and Technical Program Committee member of National Conferences and International Conferences. She has received a best researcher award during her research period. 


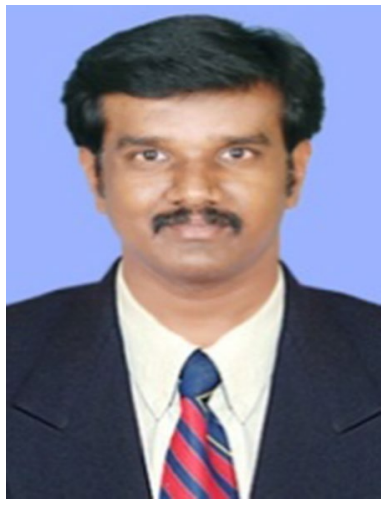

Dr. Sudhakar Sengan is currently working as Professor in the Department of Computer Science and Engineering, PSN College of Engineering and Technology, Tirunelveli-627152, Tamil Nadu, India. He received Ph.D. degree in ICE from Anna University, Chennai, Tamil Nadu, and India. He has 20 years of Experience in Teaching/Research/ Industry. He has published papers in 75 International Journals, 20 International Conferences, and 10 National Conferences. He is a Research Supervisor in Anna University in the Faculty of Information and Communication Engineering. His research interest includes Network Security, Information Security, and MANET, Cloud Computing, IoT. He received an award of Honorary Doctorate (Doctor of LettersD.LITT.) from International Economics University, SAARC Countries in the field of Education and Students Empowerment in April 2017. He has published a Textbook for Anna University syllabus: "Digital Principles and System Design" Thakur Publications Pvt. Ltd, Chennai. ISBN: 978-93-87880-77-1., Title: "Problem Solving \& Python Programming," Charulatha Publication, Chennai. Title: Operating Systems", Thakur Publications Pvt. Ltd, Chennai. ISBN-978-93-88809-15-3. He is a member of various professional bodies like MISTE, MIEEE, MIAENG, MIACSIT,MICST, and MIEDRC.

\section{Authors and Affiliations}

\section{Roy Setiawan ${ }^{1} \cdot$ Ramakoteswara Rao Ganga $^{2} \cdot$ Priya Velayutham $^{3}$. Kumaravel Thangavel ${ }^{4} \cdot$ Dilip Kumar Sharma $^{5} \cdot$ Regin Rajan $^{6}$. Sujatha Krishnamoorthy ${ }^{7}$. Sudhakar Sengan ${ }^{8}$}

Roy Setiawan

roy@petra.ac.id

Kumaravel Thangavel

kumarengineer@gmail.com

Dilip Kumar Sharma

dilipsharmajiet@gmail.com

Regin Rajan

regin12006@yahoo.co.in

Sujatha Krishnamoorthy sujatha@wku.edu.cn

1 Department Management, Universitas Kristen Petra, Surabaya, Indonesia

2 Department of Computer Science and Engineering, Koneru Lakshmaiah Education Foundation, Vaddeswaram, Andhra Pradesh 522502, India

3 Department of Computer Science and Engineering, Paavai Engineering College, Namakkal, Tamil Nadu 637018, India

4 Department of Computer Science and Engineering, Kongu Engineering College, Perundurai, Erode, Tamil Nadu, India

5 Department of Mathematics, Jaypee University of Engineering and Technology, Guna, Madhya Pradesh 473226, India

6 Department of Information Technology, Adhiyamaan College of Engineering, Hosur, Tamil Nadu 635109, India

7 Department of Computer Science, Wenzhou-Kean University, Wenzhou 325060, Zhejiang, China

8 Department of Computer Science and Engineering, PSN College of Engineering and Technology, Tirunelveli, Tamil Nadu 627152, India 